PROCEEDINGS OF THE

AMERICAN MATHEMATICAL SOCIETY

Volume 103, Number 1, May 1988

\title{
THE MIDDLE ANNIHILATOR CONJECTURE FOR EMBEDDABLE RINGS
}

\author{
C. DEAN
}

(Communicated by Donald Passman)

\begin{abstract}
It is shown that any ring which can be embedded in an Artinian ring has just finitely many middle annihilator primes. In particular, this proves the middle annihilator conjecture for a large class of Noetherian rings.
\end{abstract}

In recent years, key questions for Noetherian ring theory have dealt with the behavior of various types of prime ideals. The minimal primes, prime annihilators and affiliated primes are both useful and interesting in their own right. These ideals are all examples of middle annihilator prime ideals, and so the latter class has come under examination.

In particular, the question arises of to what extent various results for the classes of prime ideals mentioned above have analogs for middle annihilator prime ideals. The most immediate question of this sort asks whether a Noetherian ring has just finitely many middle annihilator primes. The result of this note answers this question in the affirmative for a large class of (not necessarily Noetherian) rings.

THEOREM. Let $R$ be a ring which can be embedded into an Artinian ring. Then $R$ has just finitely many middle annihilator prime ideals.

We begin by placing this result in context. Middle annihilator ideals were first defined in an unpublished note of Kaplansky.

Definition. Let $R$ be a ring with ideals $A$ and $B$ such that $A B \neq(0)$. Let $I=\{x \in R \mid A x B=0\}$. Then $I$ is the middle annihilator of $A$ and $B$; write $I=\operatorname{Mid}(A, B)$.

Note that every right annihilator ideal is a middle annihilator, and that maximal middle annihilators are prime.

Kaplansky notes that in a ring with the ascending chain condition on ideals, every minimal prime is a middle annihilator. Conversely, Krause [8] shows that a Noetherian ring admits a classical Artinian ring of quotients if and only if all of its middle annihilator primes are minimal.

When such a quotient ring exists, then the middle annihilator prime ideals are manifestly finite in number. Krause also established this result for fully bounded Noetherian rings; this has been obtained independently by Small and Stafford [11]. (The latter authors also show by example that middle annihilator primes need not be affiliated.) Goldie and Krause [4] prove that nonsingular Noethrian rings have just finitely many middle annihilator prime ideals.

Received by the editors February 10, 1987.

1980 Mathematics Subject (lassification (1985 Revision). Primary 16A33; Secondary 16A34. 
A unifying theme of the results cited here is that the rings in question admit embeddings into Artinian rings. (For FBN rings, the embedding was established in [6]; while for nonsingular Noethering rings it can be found in [2, p. 97].) Thus, the theorem given here subsumes the results mentioned above. Recently, Blair and Small [1] have shown that Krull homogenous Noetherian rings are embeddable. In addition, factors of group rings of polycyclic-by-finite groups are known to be embeddable [7], and many Noetherian rings admit ad hoc embeddings. However, the result given here is not comprehensive: While [3] shows that $U\left(\operatorname{sl}_{2}(\mathbf{C})\right)$ possesses a nonembeddable factor, in [11] it is proved that factors of enveloping algebras of finite-dimensional Lie algebras have just finitely many middle annihilator prime ideals.

THEOREM. Let $R$ be a ring which can be embedded into an Artinian ring $S$. Then $R$ has just finitely many middle annihilator prime ideals. The number of such ideals is less than or equal to the length of ${ }_{S} S$.

PROOF. Let $\left\{P_{i}\right\}$ be the set of prime middle annihilators of $R$; say $P_{i}=$ $\operatorname{Mid}\left(A_{i}, B_{i}\right)$. Then $P_{i}$ is the right annihilator of $\left(A_{i} / A_{i}^{\prime}\right)$, where $A_{i}^{\prime}$ is the left annihilator of $B_{i}$. Without loss of generality, we may assume that $A_{i}$ is the left annihilator of $P_{i} B_{i}$. Write $P_{i}=r\left(A_{i} / A_{i}^{\prime}\right)$.

Considered as an $S$ - $R$ bimodule, $S$ certainly has finite length. Note that for any $i, S A_{i} \cap R=A_{i}, S A_{i}^{\prime} \cap R=A_{i}^{\prime}$, and that $P_{i}=r\left(S A_{i} / S A_{i}^{\prime}\right)$. Thus we may pick an $S$ - $R$ bimodule composition series for $S$ which passes through $S A_{i}$ and $S A_{i}^{\prime}$, say,

$$
(0)=C_{0} \subset C_{1} \subset \cdots \subset C_{n}=S A_{i}^{\prime} \subset \cdots \subset C_{n+m}=S A_{i} \cdots C_{t}=S .
$$

For each $j \in\{1,2, \ldots, t\}$, the simplicity of $C_{j} / C_{j-1}$ as a bimodule implies that $r\left(C_{j} / C_{j-1}\right)$ is a prime ideal of $R$; call it $Q_{j}$. Now, $C_{n+m} Q_{n+m} Q_{n+m-1} \cdots Q_{n+1} \subset$ $C_{n}$; thus $Q_{n+m} Q_{n+m-1} \cdots Q_{n+1} \subset P_{i}$, and $Q_{j} \subset P_{i}$ for some $j \in\{n+1$, $n+2, \ldots, n+m\}$. The reverse inclusion being obvious, we have realized $P_{i}$ as the annihilator of a composition factor. Invoking the Jordan-Hölder Theorem, which states that the composition factors from any two series can be paired isomorphically, we see that the set $\left\{P_{i}\right\}$ is finite. Thus $R$ has just finitely many middle annihilator prime ideals. The bound on the number of these follows immediately.

The proof of this theorem is reminiscent of that of [12, Theorem 1]. In fact, using this reference we obtain further information about the middle annihilator primes of embeddable $k$-algebras ( $k$ a field) as follows. A result of Schofield [9, Theorem 7.13] asserts that every embeddable $k$-algebra can be embedded in a simple Artinian ring. Suppose that $R$ is an embeddable $k$-algebra whose prime factors are right Goldie. Let $S$ be a simple Artinian overring for $R$. Then, in the terminology of [5], the middle annihilator primes of $R$ are a subset of the (finite) set of primes affiliated to (0) in $S$; in case $S$ is simple Artinian, the primes affiliated to (0) are just the annihilators of the composition factors of the series $(*)$.

Let $X=\{$ prime ideals $P$ of $R \mid P$ is affiliated to (0) in $S\}$. Then by [12, Theorem 1], we have

$$
\operatorname{rank} S=\sum_{P \in X} n(P) \operatorname{rank}(R / P)
$$

where each $n(P)$ is a positive integer. 
However, it is not the case that the additivity formula involves only the middle annihilator primes. The following example from [10] illustrates this point.

EXAMPLE. Let $k$ be a field, $A=k\left[X_{1}, X_{2}\right]$ and define $\rho: A \rightarrow A$ by $\rho\left(X_{1}\right)=0$, $\rho\left(X_{2}\right)=X_{1}$. Let

$$
R=\left\{\left(\begin{array}{ccc}
a & b & 0 \\
X_{1} c & d & 0 \\
0 & 0 & \rho(d)
\end{array}\right) \mid a, b, c, d \in A\right\} .
$$

Then $R$ has rank $2 ; R$ can be embedded in the simple Artinian ring of $3 \times 3$ matrices over $k\left(X_{1}, X_{2}\right)$. Call this ring $S$. Now, $S$ has rank 3 ; however, the only middle annihilator prime of $R$ is (0). Thus, the additivity formula cannot be based on middle annihilator primes alone.

ACKNOWLEDGMENTS. This work forms part of the author's Ph.D. thesis. The author wishes to thank L. W. Small for his helpful comments regarding the work.

\section{REFERENCES}

1. W. D. Blair and L. W. Small, Embeddings in Artinian rings and Sylvester rank functions, Israel J. Math. 58 (1987), 10-18.

2. A. W. Chatters and C. R. Hajarnavis, Rings with chain conditions, Research Notes in Math., vol. 44, Pitman, London, 1980.

3. C. Dean and J. T. Stafford, A nonembeddable Noetherian ring, J. Algebra (in press).

4. A. W. Goldie and G. Krause, Strongly regular elements of Noetherian rings, J. Algebra 91 (1984), 410-429.

5. K. R. Goodearl and R. B. Warfield, Jr., in preparation.

6. R. Gordon, Primary decomposition in right Noetherian rings, Comm. Algebra 2 (1974), 491524.

7. A. V. Jategaonkar, Solvable Lie algebras, polycyclic-by-finite groups and bimodule Krull dimension, Comm. Algebra 10 (1982), 19-70.

8. G. Krause, Middle annihilators in Noetherian rings, Comm. Algebra 8 (1980), 781-791.

9. A. H. Schofield, Representations of rings over skew fields, London Math. Soc. Lecture Note Ser., vol. 92, Cambridge Univ. Press, London and New York, 1985.

10. L. W. Small, Rings satisfying a polynomial identity, Lecture notes, Essen, 1980.

11. L. W. Small and J. T. Stafford, Regularity of zero divisors, Proc. London Math. Soc. (3) 44 (1982), 405-419.

12. R. B. Warfield, Jr., Prime ideals in ring extensions, J. London Math. Soc. (2) 28 (1983), 453-460.

Department of Mathematics, University of Chicago, Chicago, Illinois 60637 